\title{
Demixing in athermal mixtures of colloids and excluded-volume polymers from a density functional theory
}

\author{
Paweł Bryk \\ Department for the Modeling of Physico-Chemical Processes, \\ Maria Curie-Sktodowska University, 20-031 Lublin, Poland*
}

(Dated: November 19, 2018)

\begin{abstract}
We study the structure and interfacial properties of model athermal mixtures of colloids and excluded volume polymers. The colloid particles are modeled as hard spheres whereas the polymer coils are modeled as chains formed from tangentially bonded hard spheres. Within the framework of the nonlocal density functional theory we study the influence of the chain length on the surface tension and the interfacial width. We find that the interfacial tension of the colloid-interacting polymer mixtures increases with the chain length and is significantly smaller than that of the ideal polymers. For certain parameters we find oscillations on the colloid-rich parts of the density profiles of both colloids and polymers with the oscillation period of the order of the colloid diameter. The interfacial width is few colloid diameters wide and also increases with the chain length. We find the interfacial width for the end segments to be larger than that for the middle segments and this effect is more pronounced for longer chains.
\end{abstract}

\section{INTRODUCTION}

Suspensions of sterically stabilized colloids and nonadsorbing polymers often exhibit phase separation 1, 2]. Since the experimental parameters can be tailored to match the desired properties, such systems have become an important tool in investigating various theoretical concepts. Recent experiments on well-characterized colloid-polymer mixtures have involved studies of the wetting transition [3, 4], real space observation of the thermal capillary waves [5], the fluid-fluid interfacial tension [6, 7, [8, [9], and the interfacial width [10].

One of the first theoretical models giving an insight into this phenomenon is the Asakura-Oosawa-Vrij (AOV) model of colloid-polymer mixtures 11, 12, whereby the polymer-polymer interactions are ideal but the polymer-colloid and colloid-colloid interactions are of the hard-sphere type. Demixing transition into the colloid-rich and the polymer-rich phases may be explained by invoking the concept of attractive depletion interactions that arise due to a tendency of the system to reduce the volume excluded to the centers of polymer coils [13, 14]. Brader and Evans [15] have investigated the interfacial properties of the AOV model within the square gradient approximation. Their results were in a reasonable agreement with the experimental data. Subsequently Schmidt et al. [16] have proposed an extension of Rosenfeld's successful fundamental measure theory 17] to the AOV model. It has turned out that this functional yields the interfacial tension in a good agreement with the experiment and predicts a sequence of layering transitions in the partial wetting regime prior to a transition to complete wetting [18]. These findings have been confirmed by Monte Carlo simulations [19] and recent experiments [3, 4].

The Asakura-Oosawa-Vrij model was originally introduced for systems, where the polymer coils are smaller than the colloid dimensions and the polymer-polymer interactions are neglected. The latter approximation is reasonable if the second virial coefficient of the monomer-monomer interaction vanishes what corresponds to the theta-point conditions. The influence of the polymer-polymer interactions has been studied first by Warren et al. 20] who used the perturbation theory around the AOV reference model. Fuchs and Schweizer [21, 22, 23] have noted that the polymer-polymer interactions lead to an upward shift of the spinodal. These findings have been confirmed by the results of the modified free volume theory 24], the geometry-based density functional approach [25] and sophisticated computer simulation techniques [26]. Very recently the interfacial behavior of the interacting polymer-hard sphere colloid mixtures within the square gradient approximation has been investigated 27, 28|. An important ingredient of the square gradient approximation is an expression for the direct correlation function 29]. This is usually tackled by employing the mean-spherical approximation, so that only an expression for the interaction potential is needed. Both groups have adopted different approaches. To obtain the interaction potential between two colloidal spheres mediated by excluded volume polymers Aarts et al. [27] have used the generalized Gibbs adsorption equation approach 30], whereas Moncho-Jorda et al. 28 have used a depletion potential obtained from direct computer simulations 31]. Both groups have found that the interfacial tension is lower for interacting polymers than for the AOV model.

*Electronic address: pawel@paco.umcs.lublin.pl 
In a recent work Paricaud et al. 32] have studied the phase behavior in model athermal mixtures of colloids and freely jointed tangentially bonded hard sphere polymers by means of Wertheim's first order thermodynamic perturbation theory (TPT1) 33. They have found that if the colloid diameter is much larger than the polymer segment diameter the system may undergo a demixing transition. This theory offers several advantages over previously mentioned approaches. As a fully microscopic theory it is free of the coarse-graining, which is a powerful concept on its own [34], but may be difficult to implement, for instance, close to geometrically structured substrates [35, 36]. The two-body potentials resulting from the coarse-graining techniques may be insufficient to correctly describe the underlying complex system and the higher-order terms may have to be taken into account [37]. Also, within the TPT1 theory it is straightforward to incorporate the attractive interactions. However, the Wertheim TPT1 description is not free of its own caveats. For example, an extension of the theory to the solid phase while in principle possible 38], it is difficult to carry out for the colloid-polymer mixtures. Within the TPT1 theory the second virial coefficient scales linearly with the chain length $M[39,40]$ instead of $\sim M^{3 \nu}$, where $\nu$ is the Flory exponent. The consequence is that within the TPT1 treatment the dilute regime is not described accurately. Although it is possible to improve this deficiency [41], it is not clear how this approach could be extended to the colloid-polymer mixtures. Despite these issues, the approach of Paricaud et al. [32] seems to be quite interesting.

In the present work we apply the microscopic density functional theory of $\mathrm{Yu}$ and $\mathrm{Wu}$ 42] to study the interfacial properties of the hard sphere colloids and excluded volume polymers modeled as freely jointed tangentially bonded hard spheres. In the bulk limit this functional yields an equation of state identical with that of Ref. [32. The applied functional is nonlocal, therefore it should correctly capture the structure of the interface including possible oscillatory behavior of the density profiles. Also, the Yu and Wu theory treats the polymer chains and colloid spheres on equal footing, therefore the many-body effects are automatically included.

\section{THEORY}

In this work we consider mixtures of colloids (species $C$ ) and polymers (species $P$ ). The colloidal particles are represented as hard spheres of diameter $\sigma_{C}$ whereas the polymer coils are modeled as chains built of $M$ tangentially bonded hard-sphere segments of diameter $\sigma_{P S}$. We assume that there are no torsional or bending potentials imposed on the polymer segments, i.e. the monomers are freely jointed and any arbitrary polymer configuration free of the intermolecular and intramolecular overlaps is allowed. By introducing the total bonding potential $V_{b}(\mathbf{R})$ as a sum of bonding potentials $v_{b}$ between the monomers $V_{b}(\mathbf{R})=\sum_{i=1}^{M-1} v_{b}\left(\left|\mathbf{r}_{i+1}-\mathbf{r}_{i}\right|\right)$ the intramolecular interactions for the freely-jointed tangential hard spheres can be conveniently written as

$$
\exp \left[-\beta V_{b}(\mathbf{R})\right]=\prod_{i=1}^{M-1} \frac{\delta\left(\left|\mathbf{r}_{i+1}-\mathbf{r}_{i}\right|-\sigma_{P S}\right)}{4 \pi \sigma_{P S}^{2}} .
$$

In the above $\mathbf{R} \equiv\left(\mathbf{r}_{1}, \mathbf{r}_{2}, \cdots, \mathbf{r}_{M}\right)$ denotes a set of monomer coordinates.

As in every density functional theory the grand potential of the system is assumed to be a functional of the local densities of polymers $\rho_{P}(\mathbf{R})$ and colloids $\rho_{C}(\mathbf{r})$

$$
\begin{gathered}
\Omega\left[\rho_{P}(\mathbf{R}), \rho_{C}(\mathbf{r})\right]=F\left[\rho_{P}(\mathbf{R}), \rho_{C}(\mathbf{r})\right]+ \\
\int d \mathbf{R} \rho_{P}(\mathbf{R})\left(V_{\text {ext }}^{(P)}(\mathbf{R})-\mu_{P}\right) \\
+\int d \mathbf{r} \rho_{C}(\mathbf{r})\left(V_{\text {ext }}^{(C)}(\mathbf{r})-\mu_{C}\right)
\end{gathered}
$$

where $V_{e x t}^{(P)}(\mathbf{R}), \mu_{P}, V_{e x t}^{(C)}(\mathbf{r})$ and $\mu_{C}$ are the external and the chemical potentials for polymers and colloids, respectively. The free energy of the system $F$ is a sum of the ideal and excess contributions, $F=F_{i d}+F_{e x}$. The ideal part of the free energy is known exactly

$$
\begin{aligned}
& \beta F_{i d}\left[\rho_{P}(\mathbf{R}), \rho_{C}(\mathbf{r})\right]=\beta \int d \mathbf{R} \rho_{P}(\mathbf{R}) V_{b}(\mathbf{R}) \\
& +\int d \mathbf{R} \rho_{P}(\mathbf{R})\left[\ln \left(\rho_{P}(\mathbf{R})\right)-1\right] \\
& +\int d \mathbf{r} \rho_{C}(\mathbf{r})\left[\ln \left(\rho_{C}(\mathbf{r})\right)-1\right],
\end{aligned}
$$

where the dependence on the irrelevant thermal wavelengths for polymers and colloids was suppressed. 
In their study of bulk properties of athermal mixtures of colloid and excluded-volume polymers Paricaud et al. 32 . employed the first order thermodynamic perturbation theory of Wertheim [33. Within this approach the excess free energy of the polymer system can be treated as a sum of the excess free energy of the reference system containing unbonded monomers and a perturbation due to formation of the polymer coils. Yu and Wu [42] incorporated Wertheim's first-order perturbation theory into the framework of the fundamental measure theory (FMT) of Rosenfeld [17] providing thus a convenient mean of description of inhomogeneous mixtures of tangentially jointed hard-sphere chains. The connection between TPT1 and FMT can be made by assuming that $F_{e x}$ is a functional of the local density of colloids and average segment densities [42] $\rho_{P S}(\mathbf{r})$ defined as

$$
\rho_{P S}(\mathbf{r})=\sum_{i=1}^{M} \rho_{P S, i}(\mathbf{r})=\sum_{i=1}^{M} \int d \mathbf{R} \delta\left(\mathbf{r}-\mathbf{r}_{i}\right) \rho_{P}(\mathbf{R})
$$

where $\rho_{P S, i}(\mathbf{r})$ is the local density of the polymer segment $i$. The convolutions of the local densities $\rho_{j}(\mathbf{r}), j=P S, C$ with a suitable set of weight functions $w_{\alpha}^{(j)}(\mathbf{r}), \alpha=3,2,1,0, V 2, V 1$ yield the weighted densities $n_{\alpha}=n_{\alpha}^{(P S)}+n_{\alpha}^{(C)}$

$$
n_{\alpha}^{(j)}(\mathbf{r})=\int d \mathbf{r}^{\prime} \rho_{j}\left(\mathbf{r}^{\prime}\right) w_{\alpha}^{(j)}\left(\mathbf{r}-\mathbf{r}^{\prime}\right)
$$

The weight functions $w_{\alpha}^{(j)}(\mathbf{r})$ are related to the geometric properties of colloidal particles and monomer segments [17]

$$
\begin{gathered}
w_{3}^{(j)}(\mathbf{r})=\Theta\left(\frac{\sigma_{j}}{2}-|\mathbf{r}|\right) \quad, \quad w_{2}^{(j)}(\mathbf{r})=\delta\left(\frac{\sigma_{j}}{2}-|\mathbf{r}|\right), \\
\boldsymbol{w}_{V 2}^{(j)}(\mathbf{r})=\frac{\mathbf{r}}{|\mathbf{r}|} \delta\left(\frac{\sigma_{j}}{2}-|\mathbf{r}|\right) \quad, w_{1}^{(j)}(\mathbf{r})=\frac{w_{2}^{(j)}(\mathbf{r})}{2 \pi \sigma_{j}}, \\
w_{0}^{(j)}(\mathbf{r})=\frac{w_{2}^{(j)}(\mathbf{r})}{\pi \sigma_{j}^{2}} \quad, \boldsymbol{w}_{V 1}^{(j)}(\mathbf{r})=\frac{\boldsymbol{w}_{V 2}^{(j)}(\mathbf{r})}{2 \pi \sigma_{j}} .
\end{gathered}
$$

In the FMT approach the excess free energy is obtained as a volume integral over the free energy density $F_{e x}=\int d \mathbf{r} \Phi$ expressed as a simple function of the weighted densities. Following $\mathrm{Yu}$ and $\mathrm{Wu}[42]$ we assume that $\Phi=\Phi_{P}+\Phi_{H S}$, where $\Phi_{H S}$ describes the reference mixture of hard spheres of diameters $\sigma_{C}$ and $\sigma_{P S}$, while the excess free energy density due to the chain connectivity $\Phi_{P}$ is an "inhomogeneous counterpart" of the perturbation term in TPT1.

There are several expressions for the hard-sphere part $\Phi_{H S}$. For the present problem we choose the elegant and inspiring White-Bear version of the FMT [43, 44]

$$
\begin{gathered}
\Phi_{H S}\left(\left\{n_{\alpha}\right\}\right)=-n_{0} \ln \left(1-n_{3}\right)+\frac{n_{1} n_{2}-\boldsymbol{n}_{V 1} \cdot \boldsymbol{n}_{V 2}}{1-n_{3}} \\
+\left(n_{2}^{3}-3 n_{2} \boldsymbol{n}_{V 2} \cdot \boldsymbol{n}_{V 2}\right) \frac{n_{3}+\left(1-n_{3}\right)^{2} \ln \left(1-n_{3}\right)}{36 \pi\left(n_{3}\right)^{2}\left(1-n_{3}\right)^{2}}
\end{gathered}
$$

The contribution $\Phi_{P}$ due to chain connectivity is evaluated using Wertheim's first-order perturbation theory 33 , 42

$$
\Phi_{P}\left(\left\{n_{\alpha}\right\} ;\left\{n_{\alpha}^{(P S)}\right\} ; \sigma_{P S}\right)=\frac{1-M}{M} n_{0}^{(P S)} \zeta^{(P S)} \ln \left[y_{H S}\left(\sigma_{P S} ;\left\{n_{\alpha}\right\}\right)\right],
$$

where $\zeta^{(P S)}=1-\mathbf{n}_{V 2}^{(P S)} \cdot \mathbf{n}_{V 2}^{(P S)} /\left(n_{2}^{(P S)}\right)^{2} \cdot y_{H S}$ is the Boublik-Mansoori-Carnahan-Starling-Leland [45, 46] expression for the contact value of the radial distribution function of mixtures hard spheres

$$
y_{H S}\left(\sigma_{P S} ;\left\{n_{\alpha}\right\}\right)=\frac{1}{1-n_{3}}+\frac{n_{2} \sigma_{P S} \zeta}{4\left(1-n_{3}\right)^{2}}+\frac{\left(n_{2} \sigma_{P S}\right)^{2} \zeta}{72\left(1-n_{3}\right)^{3}} .
$$

with $\zeta=1-\mathbf{n}_{V 2} \cdot \mathbf{n}_{V 2} /\left(n_{2}\right)^{2}$.

At equilibrium the first functional derivative of the grand potential with respect to the densities of colloids and polymers vanishes

$$
\frac{\delta \Omega\left[\rho_{P}(\mathbf{R}), \rho_{C}(\mathbf{r})\right]}{\delta \rho_{P}(\mathbf{R})}=\frac{\delta \Omega\left[\rho_{P}(\mathbf{R}), \rho_{C}(\mathbf{r})\right]}{\delta \rho_{C}(\mathbf{r})}=0
$$


which leads to the following equation for the average segment density profile

$$
\rho_{P S}(\mathbf{r})=\exp \left(\beta \mu_{P}\right) \int d \mathbf{R} \sum_{i=1}^{M} \delta\left(\mathbf{r}-\mathbf{r}_{i}\right) \exp \left[-\beta V_{b}(\mathbf{R})-\beta \sum_{l=1}^{M} \lambda_{l}\left(\mathbf{r}_{l}\right)\right]
$$

where $\lambda_{l}\left(\mathbf{r}_{l}\right)$ is

$$
\lambda_{l}\left(\mathbf{r}_{l}\right)=\frac{\delta F_{e x}}{\delta \rho_{P S}\left(\mathbf{r}_{l}\right)}+v_{l}\left(\mathbf{r}_{l}\right)
$$

with $v_{l}\left(\mathbf{r}_{l}\right)$ being an external potential acting on the $l$-th segment. Equation (13) can be rewritten as

$$
\rho_{P S}(\mathbf{r})=\exp \left(\beta \mu_{P}\right) \sum_{i=1}^{M} \exp \left[-\beta \lambda_{i}(\mathbf{r})\right] G_{i}(\mathbf{r}) G_{M+1-i}(\mathbf{r}),
$$

where the propagator function $G_{i}(\mathbf{r})$ is determined from the recurrence relation

$$
G_{i}(\mathbf{r})=\int d \mathbf{r}^{\prime} \exp \left[-\beta \lambda_{i}\left(\mathbf{r}^{\prime}\right)\right] \frac{\delta\left(\sigma_{P S}-\left|\mathbf{r}-\mathbf{r}^{\prime}\right|\right)}{4 \pi \sigma_{P S}^{2}} G_{j-1}\left(\mathbf{r}^{\prime}\right)
$$

for $i=2,3, \ldots, M$ and with $G_{1}(\mathbf{r}) \equiv 1$. Likewise Eq. (12) leads to the following equation for the density profile of colloids

$$
\rho_{C}(\mathbf{r})=\exp \left[\beta \mu_{C}-\beta V_{e x t}^{(C)}(\mathbf{r})-\beta \delta F_{e x} / \delta \rho_{C}(\mathbf{r})\right]
$$

Equations (15) and (17) can be solved numerically using the standard Picard iterative method.

\section{RESULTS}

In homogeneous systems the thermodynamic properties can be calculated analytically. To this end we note that in the bulk the vector weighted densities vanish whereas the scalar weighted densities become proportional to the corresponding bulk densities. For the polymer weighted densities we have $n_{\alpha}^{(P S)}=\xi_{\alpha}^{(P)} M \rho_{P}^{(b)}=\xi_{\alpha}^{(P)} \rho_{P S}^{(b)}$, with $\xi_{3}^{(P)}=\pi / 6 \sigma_{P S}^{3}, \xi_{2}^{(P)}=\pi \sigma_{P S}^{2}, \xi_{1}^{(P)}=\sigma_{P S} / 2$ and $\xi_{0}^{(P)}=1$. Similar, for the colloid weighted densities we have $n_{\alpha}^{(C)}=$ $\xi_{\alpha}^{(C)} \rho_{C}^{(b)}$. The total configurational free energy per unit volume, $\Phi_{v}$, can be straightforwardly obtained by inserting the expressions for the weighted densities into Eqs. [(9)-(11)], $\Phi_{v}=\Phi_{H S}+\Phi_{P}+\beta^{-1} \rho_{P}^{(b)}\left[\ln \left(\rho_{P}^{(b)}\right)-1\right]+\beta^{-1} \rho_{C}^{(b)}\left[\ln \left(\rho_{C}^{(b)}\right)-1\right]$. Finally, the pressure $P$, and the chemical potentials of both species are determined from

$$
\beta P=-\Phi_{v}+\sum_{j=P, C} \rho_{j}^{(b)} \frac{\partial \Phi_{v}}{\partial \rho_{j}^{(b)}}, \beta \mu_{j}=\frac{\partial \Phi_{v}}{\partial \rho_{j}^{(b)}}
$$

For bulk systems our theory is identical to the Wertheim TPT1 theory for athermal mixtures of hard spheres and excluded-volume polymers. Paricaud et al. 32] conjectured that TPT1 predicts a demixing transition into colloid-rich (polymer-poor) and colloid-poor (polymer-rich) phases. The coexisting equilibrium densities (binodals) were obtained from the condition of the equality of pressure and chemical potentials of both species in the two demixed phases. The spinodal lines delimiting the regions of the stability against fluctuations of density and composition were calculated from $\operatorname{det}\left[\partial^{2} \Phi_{v} / \partial \rho_{i}^{(b)} \partial \rho_{j}^{(b)}\right]=0, i, j=P, C$. The critical points were evaluated from [47]

$$
\begin{gathered}
s^{3} \frac{\partial^{3} \Phi_{v}}{\partial\left[\rho_{P}^{(b)}\right]^{3}}+3 s^{2} \frac{\partial^{3} \Phi_{v}}{\partial\left[\rho_{P}^{(b)}\right]^{2} \partial \rho_{C}^{(b)}}+3 s \frac{\partial^{3} \Phi_{v}}{\partial \rho_{P}^{(b)} \partial\left[\rho_{C}^{(b)}\right]^{2}}+\frac{\partial^{3} \Phi_{v}}{\partial\left[\rho_{C}^{(b)}\right]^{3}}=0 \\
s \equiv \frac{-\partial^{2} \Phi_{v}}{\partial \rho_{P}^{(b)} \partial \rho_{C}^{(b)}} / \frac{\partial^{2} \Phi_{v}}{\partial\left[\rho_{P}^{(b)}\right]^{2}} .
\end{gathered}
$$

Figure 1 shows the fluid-fluid phase equilibria (solid lines), the spinodals (dashed lines) and the critical points (black dots) for model athermal mixtures of colloids and excluded-volume polymers considered in this work. The calculations were carried out for the constant colloid to polymer segment size ratio $d=\sigma_{C} / \sigma_{P S}=10$ and for $M=80$, 
100, 120, 200 and 500, from top to bottom, respectively. The diagrams are plotted in the colloid packing fraction - reduced pressure representation, which will be useful in the presentation of the numerical results described below. Note that the reciprocal of the reduced pressure plays the role similar to the temperature in the case of simple fluids, consequently the tie lines connecting the coexisting fluid states are horizontal. The Wertheim TPT1 theory leads to the incorrect Flory exponent $(\nu=0.5$ rather than 0.588$)$ therefore the comparison in terms of the gyration radius to colloid radius size ratio $q=R_{g} / R_{C}$ is not entirely meaningful but on the other hand, $R_{g}$ does not enter explicitly the numerical calculations at any point. Therefore, assuming that the polymer gyration radius scales as $R_{g}=a_{P} M^{\nu} \sigma_{P S}$ with $a_{P} \approx 0.5$ 41, 48, 49], our systems would correspond to $q=0.894,1,1.095,1.414$, and 2.236 for $M=80,100$, 120, 200, and 500, respectively. Thus we consider both regimes $q<1$ and $q>1$. We note that the reduced critical pressure $\beta P^{(c r)} \sigma_{C}^{3}$ and the critical colloid packing fraction $\eta_{C}^{(c r)}$ decrease as the chain length increases. However, as mentioned in Ref. [32], for $M \rightarrow \infty$ and for fixed $d$ both $\beta P_{\infty}^{(c r)} \sigma_{C}^{3}$ and $\eta_{C, \infty}^{(c r)}$ tend to a finite, nonzero value. For $d=10$ we find that $\beta P_{\infty}^{(c r)} \sigma_{C}^{3}=0.725456$ and $\eta_{C, \infty}^{(c r)}=0.176477$ with the leading order correction $\sim \mathcal{O}(1 / \sqrt{M})$. This result is in a qualitative agreement with the phase behavior in the so-called "protein limit" of colloid-polymer mixtures $50,51,52]$.

We turn now to the interface between the demixed fluid phases. In Figs. 2 and 3 we display the representative examples of the colloid and average polymer segment density profiles evaluated for $M=200$ and for the reduced pressures $\beta P \sigma_{C}^{3}=7(\mathrm{~A}), 6(\mathrm{~B}), 4(\mathrm{C})$ and $3.58(\mathrm{D})$. The profiles close to the critical point are diffuse and smooth. However for larger pressures oscillations develop on the colloid-rich side of the profiles (see the insets). It is interesting to note that the consecutive maxima of these oscillations are located at distances approximately equal the colloid diameter. The presence of the oscillations on both profiles can be explained by invoking the theory of the asymptotic decay of the correlation functions in simple fluids [53]. However, here the polymer is represented as a chain consisting of 200 monomers and it is not clear how the arguments of Ref. [53] can be extended to complex fluids. Nevertheless it is good to see that the results from the present work are qualitatively similar to the findings of Brader et al. 18 .

Figure 4 shows the reduced interfacial tension $\beta \gamma \sigma_{C}^{2}$ as a function of the order parameter $\Delta \eta_{C}$ i.e. the difference in the colloid packing fractions in coexisting "liquid" (colloid-rich) and vapor (colloid-poor) phases evaluated for the chain lengths $M=80$ (solid line), 120 (dashed line), 200 (dotted line) and 500 (dot-dashed line). We find that the surface tension increases with the chain length. Note that the reduced surface tension is significantly smaller than that of ideal polymers. These findings are in accordance with the recent results obtained from the squar gradient approximation [27, 28].

In Fig. [5 we examine the critical behavior of the interfacial tension resulting from the present theory. Part a shows the log-log plot of the interfacial tension vs. the order parameter $\Delta \eta_{C}$ whereas part b displays the logarithm of the interfacial tension plotted against the logarithm of the relative distance to the critical point measured in terms of $\left(P-P^{(c r)}\right) / P^{(c r)}$ (i.e. the analog of the reduced temperature $\tau=\left(T^{(c r)}-T\right) / T^{(c r)}$ for simple fluids). We find that on approaching the critical point the surface tension vanishes with classical (mean field) exponents. This is not surprising in view of the fact that the Wertheim TPT1 theory can be derived by considering the chemical potential of a single chain in the reference solvent of monomers 32, 54]. Similar results have been obtained in a related study of binary polymer blends [55].

The width of the interface can be quantitatively characterized by the parameter $W$, defined as [56]

$$
W=\left[\rho_{P S}(z=\infty)-\rho_{P S}(z=-\infty)\right]\left[\frac{d \rho_{P S}(z)}{d z}\right]_{z=z_{0}}^{-1}
$$

where $z_{0}$ is given by $\rho_{P S}\left(z_{0}\right)=(1 / 2)\left[\rho_{P S}(z=\infty)+\rho_{s}(z=-\infty)\right]$ and $\rho_{P S}(z=\infty)$ and $\rho_{P S}(z=-\infty)$ are the segment densities of the coexisting polymer-rich and polymer-poor phases. In Fig. 6] we plot the interfacial width calculated for the systems with $M=80$ (solid line), 200 (dashed line) and 500 (dot-dashed line). Similar to other studies 28], we find that the interfacial width increases with the chain length. For the states well removed from the critical point it is of the order of two colloid diameters. On approaching the critical point $W$ diverges as expected. In simple fluids the interfacial width should behave similar to the correlation length $\xi$ i.e. $W \sim \xi \sim \tau^{-\nu}$. The inset illustrates that in our approach the interfacial width indeed diverges with the mean field exponent $\nu=1 / 2$, what is consistent with the surface tension results.

Finally we address the issue of the distribution of the particular segments across the interface. Several authors have pointed out that the fluid-fluid interface in polymeric systems is enriched in end-segments [57, 58, 59]. It is related to a different chain length dependence of surface tension from bulk properties [60]. Figure 7 shows the middle (solid lines) and end segment density profiles for the systems with $M=200$ and for the reduced pressures $\beta P \sigma_{c}^{3}=7(\mathrm{~A})$, $4.5(\mathrm{E})$ and $3.85(\mathrm{D})$. For the system well removed from the critical point [marked as (A)], one observes a visible difference between the segment profiles. The interface of the middle segments is sharper whereas the interface of the end segments is more diffuse. For the intermediate value of the pressure [system (E)] the difference in the distribution becomes less noticeable, and in the vicinity of the critical point [system (D)] the distributions of the middle and end 
segments are practically identical. This behavior is more pronounced for longer chains, as it is clearly visible in Figure 8 where we plot the interfacial width of the middle and end segments evaluated for the chains with $M=80$ (solid and dotted lines) and for the longest chains considered in this work, $M=500$ (dashed and dot-dashed lines). For states far away from the critical point the difference of the interfacial width for $M=80$ is around one polymer segment diameter while for $M=500$ is around the colloid radius. We conclude that for still longer chains the difference in the distribution of the middle and end segments may be as large as colloid diameter which in turn should be significant since the interfacial width of the average polymer segment is of the order of 2-3 colloid diameters. This finding cannot be ascertained if the coarse-grained approach to the description of the polymer is employed.

\section{CONCLUSIONS}

We have studied the interfacial properties of model athermal mixtures of colloids and excluded volume polymers. The colloid particles are modeled as hard spheres while the polymer coils are modeled as freely jointed tangentially bonded hard sphere chains. Model systems have been studied within the framework of the nonlocal density functional theory for the fixed ratio of the colloid to polymer segment diameters and for different chain lengths. We have found that the interfacial tension of the interacting polymer-colloid mixtures increases with the chain length and is significantly smaller than that of the ideal polymers. These results are in accordance with the recent studies obtained within the square gradient approach 27, 28]. For certain parameters we find oscillations on the colloid-rich parts of the density profiles of both colloids and polymers with the oscillation period of the order of the colloid diameter. The interfacial width is few colloid diameters wide and also increases with the chain length. We find the interfacial width for the end segments to be larger than that for the middle segments and this effect is more pronounced for longer chains. Within our approach the interfacial properties close to the critical point behave in a mean field fashion.

Before the quantitative comparison with experiments is possible some important the issues associated with the framework proposed by Paricaud et al. [32] should be solved. In particular, further research should focus on tackling the problem of the inaccurate description of the polymer in the dilute limit and the incorporation of the fluid-solid equilibria into the framework. However, despite these deficiencies the microscopic model of the colloids-excluded volume polymer mixtures studied in the present work seems to be an interesting alternative to the coarse-grained approaches.

\section{Acknowledgments}

This work has been supported by KBN of Poland under the Grant 1P03B03326 (years 2004-2006).

[1] R. Tuinier, J. Rieger, and C.G. de Kruif, Adv. Colloid Interface Sci. 103, 1 (2003).

[2] W.C.K. Poon, J. Phys.: Condens. Matter 14, R859 (2002).

[3] W.K. Wijting, N.A.M. Besseling, and M.A. Cohen Stuart, Phys. Rev. Lett. 90, 196101 (2003).

[4] W.K. Wijting, N.A.M. Besseling, and M.A. Cohen Stuart, J. Phys. Chem. B 107, 10565 (2003).

[5] D.A.G.L. Aarts, M. Schmidt, and H.N.W. Lekkerkerker, Science 304, 847 (2004).

[6] D.A.G.L. Aarts, J.H. van der Wiel, and H.N.W. Lekkerkerker, J. Phys.: Condens. Matter 15, S245 (2003).

[7] E.H.A. de Hoog and H.N.W. Lekkerkerker, J. Phys. Chem. B 103, 5274 (1999).

[8] B.-H. Chen, B. Payandeh, and M. Robert, Phys. Rev. E 62, 2369 (2000).

[9] B.-H. Chen, B. Payandeh, and M. Robert, Phys. Rev. E 64, 042401 (2001).

[10] E.H.A. de Hoog, H.N.W. Lekkerkerker, J. Schulz, and G.H. Findenegg, J. Phys. Chem. B 103, 10657 (1999).

[11] S. Asakura and F. Oosawa, J. Chem. Phys. 22, 1255 (1954).

[12] A. Vrij, Pure Appl. Chem. 48, 471 (1976).

[13] A.P. Gast, C.K. Hall, and W.B. Russell, J. Colloid Interface Sci. 96, 251 (1983).

[14] H.N.W. Lekkerkerker, W.C.K. Poon, P.N. Pusey, A. Stroobants, and P.B. Warren, Europhys. Lett. 20, 559 (1992).

[15] J.M. Brader and R. Evans, Europhys. Lett. 49, 678 (2000).

[16] M. Schmidt, H. Löwen, J.M. Brader, and R. Evans, Phys. Rev. Lett. 85, 1934 (2000).

[17] Y. Rosenfeld, Phys. Rev. Lett. 63, 980 (1989).

[18] J.M. Brader, R. Evans, M. Schmidt, and H. Löwen, J. Phys.: Condens. Matter 14, L1 (2002).

[19] M. Dijkstra and R. van Roij, Phys. Rev. Lett. 89, 208303 (2002).

[20] P.B. Warren, S.M. Ilett, and W.C.K. Poon, Phys. Rev. E 52, 5205 (1995).

[21] M. Fuchs and K.S. Schweizer, Europhys. Lett. 51, 621 (2000).

[22] M. Fuchs and K.S. Schweizer, Phys. Rev. E 64, 21514 (2002). 
[23] M. Fuchs and K.S. Schweizer, J. Phys.: Condens. Matter 14, R239 (2002).

[24] D.A.G.L. Aarts, R. Tuinier, and H.N.W. Lekkerkerker, J. Phys.: Condens. Matter 14, 7551 (2002).

[25] M. Schmidt, A.R. Denton, and J.M. Brader, J. Chem. Phys. 118, 1541 (2003).

[26] P.G. Boluis, A.A. Louis, and J.-P. Hansen, Phys. Rev. Lett. 89, 128302 (2002).

[27] D.A.G.L. Aarts, R.P.A. Dullens, H.N.W. Lekkerkerker, D. Bonn, and R. van Roij, J. Chem. Phys. 120, 1973 (2004).

[28] A. Moncho-Jorda, B. Rotenberg, and A.A. Louis, J. Chem. Phys. 119, 12667 (2003).

[29] R. Evans, in Fundamentals of Inhomogeneous Fluids, edited by D. Henderson (Dekker, New York, 1992 ), p. 85.

[30] R. Tuinier, H.N.W. Lekkerkerker, and D.A.G.L. Aarts, Phys. Rev. E 65, 060801 (2002).

[31] A.A. Louis, P.G. Bolhuis, E.J. Meijer, and J.-P. Hansen, J. Chem. Phys. 117, 1893 (2002).

[32] P. Paricaud, S. Varga, and G. Jackson, J. Chem. Phys. 118, 8525 (2003).

[33] M.S. Wertheim, J. Chem. Phys. 87, 7323 (1987).

[34] A.A. Louis, P.G. Bolhuis, J.-P. Hansen, and E.J. Meijer, Phys. Rev. Lett. 85, 2522 (2000).

[35] P. Bryk, R. Roth, M. Schoen, and S. Dietrich, Europhys. Lett. 63, 233 (2003).

[36] W. Li and H.R. Ma, Eur. Phys. J. E 12, 321 (2003).

[37] A. Moncho-Jorda, A.A. Louis, P.G. Bolhuis, and R. Roth, J. Phys.: Condens. Matter 15, S3429 (2003).

[38] C. Vega and L.G. MacDowell, J. Chem. Phys. 114, 10411 (2001).

[39] T. Boublik, Mol. Phys. 68, 191 (1989).

[40] T. Boublik, C. Vega, and M. Diaz Pena, J. Chem. Phys. 93, 730 (1990).

[41] C. Vega, J.M. Labaig, L.G. MacDowell, and E. Sanz, J. Chem. Phys. 113, 10398 (2000).

[42] Y.-X. Yu and J. Wu, J. Chem. Phys. 117, 2368 (2002).

[43] R. Roth, R. Evans, A. Lang, and G. Kahl, J. Phys.: Condens. Matter 14, 12063 (2002).

[44] Y.-X. Yu and J. Wu, J. Chem. Phys. 117, 10165 (2002).

[45] T. Boublik, J. Chem. Phys. 53, 471 (1970).

[46] G.A. Mansoori, N.F. Carnahan, K.E. Starling, and T.W. Leland, J. Chem. Phys. 54, 1523 (1971).

[47] J.S. Rowlinson, Liquids and Liquid Mixtures, (Butterworths, London, 1959), p. 205.

[48] J. Dautenhahn and C.K. Hall, Macromolecules 27, 5399 (1994).

[49] L. Lue, J. Chem. Phys. 112, 3422 (2000).

[50] P. Bolhuis, E.J. Meijer, and A.A. Louis, Phys. Rev. Lett. 90, 68304 (2003).

[51] R.P. Sear, Phys. Rev. E 66, 51401 (2002).

[52] P. Bryk, Phys. Rev. E 68, 62501 (2003).

[53] R. Evans, R.J.F. Leote de Carvalho, J.R. Henderson, and D.C. Hoyle J. Chem. Phys. 100, 591 (1994).

[54] A. Ben-Naim, J. Chem. Phys. 54, 1387 (1971).

[55] P. Bryk and S. Sokołowski, J. Chem. Phys. 120, 8299 (2004).

[56] J. Fischer and M. Methfessel, Phys. Rev. A 22, 2836 (1980).

[57] G.J.A. Ypma, P. Cifra, E. Nies, and A.R.D. van Bergen, Macromolecules 29, 1252 (1996).

[58] S.K. Kumar, M. Vacatello, and D.Y. Yoon, Macromolecules 23, 2189 (1990).

[59] J. Reiter, G. Zifferer, and O.F. Olaj, Macromolecules 23, 224 (1990).

[60] I. Szleifer and B. Widom, J. Chem. Phys. 90, 7524 (1989). 


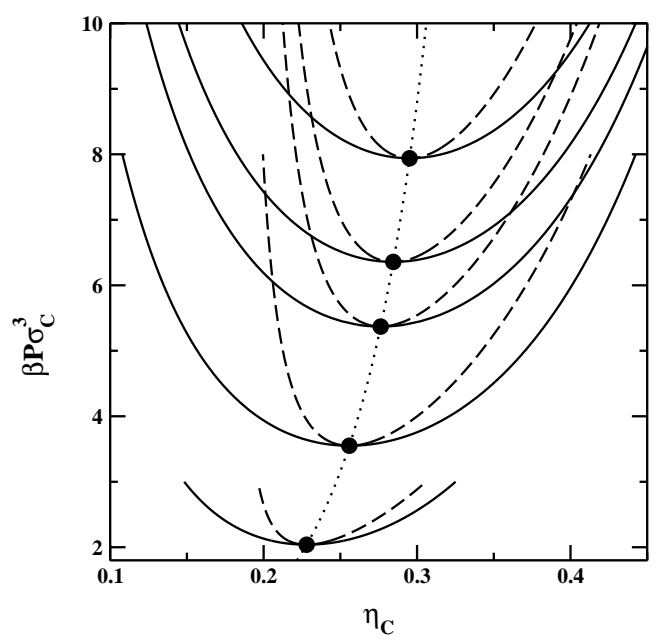

FIG. 1: The binodals (solid lines), the spinodals (dashed lines) and the critical points (black dots) for the demixing transition in colloid-excluded volume polymer mixtures resulting from the TPT1 theory. The phase diagrams are evaluated for the constant ratio of colloid diameter to polymer segment diameter $d=\sigma_{C} / \sigma_{P S}=10$ and for $M=80,100,120,200$ and 500 from top to bottom, respectively. The dotted line marks the line of critical points of the demixing transition for different chain lengths.

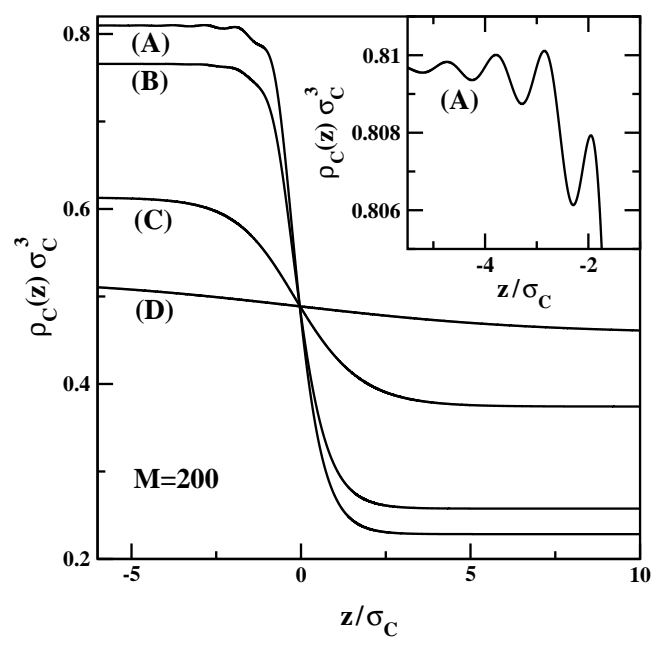

FIG. 2: Colloid density profiles across the liquid-liquid interface calculated for the system with $d=10$ and $M=200$. The profiles are evaluated for the reduced pressures $\beta P \sigma_{C}^{3}=7(\mathrm{~A}), 6(\mathrm{~B}), 4(\mathrm{C})$ and $3.58(\mathrm{D})$. The inset shows an enlargement of the profile 


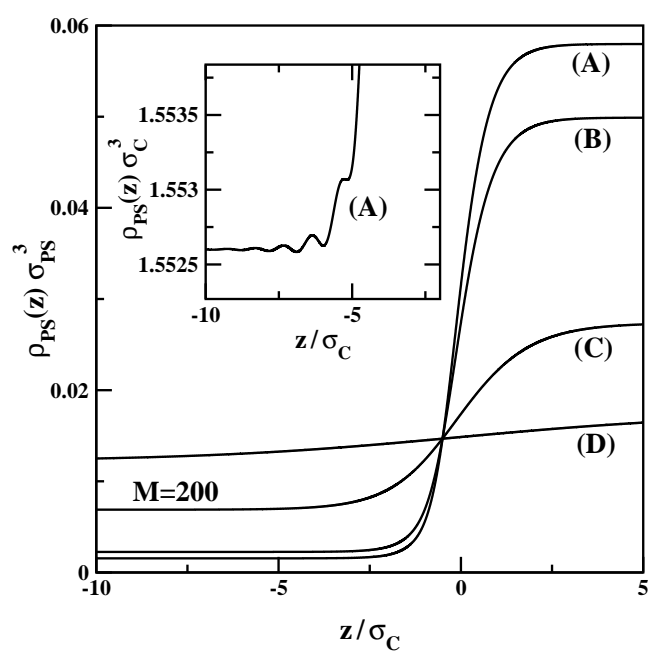

FIG. 3: Average polymer segment density profiles across the liquid-liquid interface calculated for the system with $d=10$ and $M=200$. The profiles are evaluated for the reduced pressures $\beta P \sigma_{C}^{3}=7(\mathrm{~A}), 6(\mathrm{~B}), 4(\mathrm{C})$ and $3.58(\mathrm{D})$.

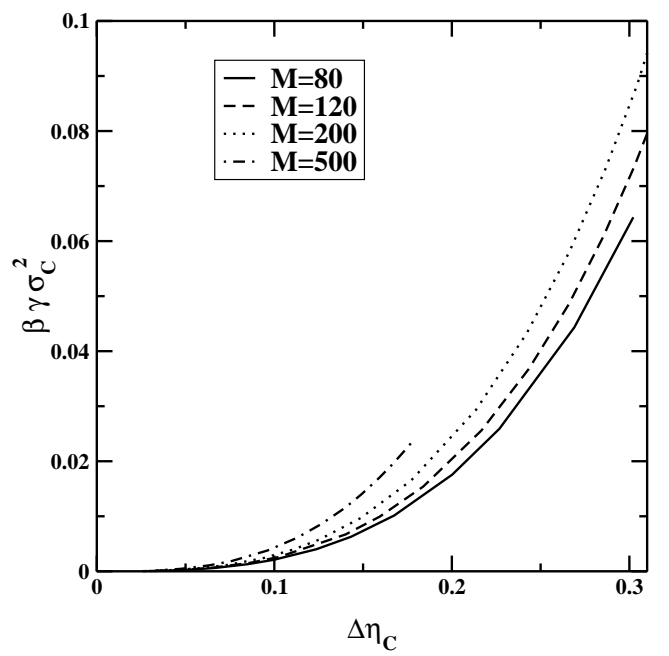

FIG. 4: The reduced surface tension $\beta \gamma \sigma_{C}^{2}$ plotted against the difference in the colloid packing fractions in the coexisting liquid (colloid-rich, L) and vapor (colloid-poor, V) phases, $\Delta \eta_{C}=\eta_{C}^{(L)}-\eta_{C}^{(V)}$. The chain lengths are given in the Figure. 

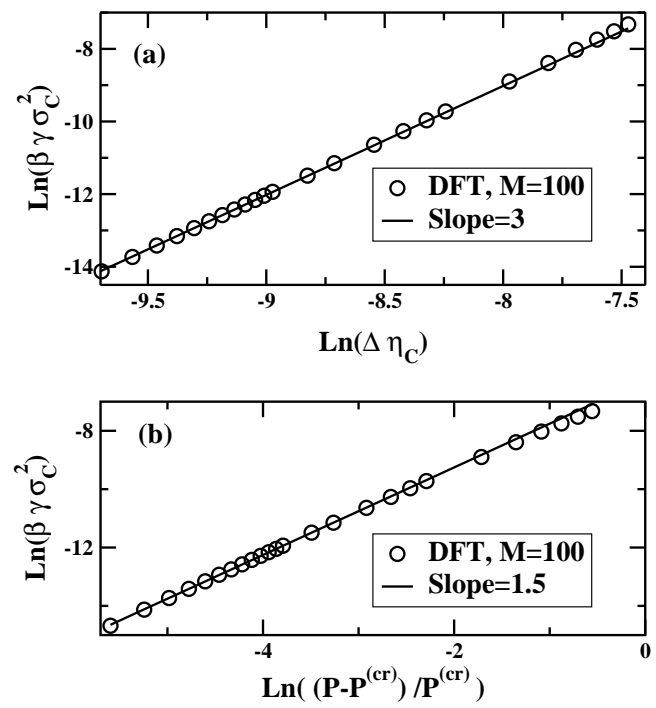

FIG. 5: Critical behavior of the reduced surface tension of colloids with $d=10$ and excluded-volume polymers with $M=100$. The open circles denote the DFT results whereas the solid lines have slopes corresponding to the classical (mean-field) critical exponents. (a) The logarithm of the reduced surface tension $\beta \gamma \sigma_{C}^{2}$ plotted against the logarithm of the difference in the colloid packing fractions in the coexisting liquid (colloid-rich, L) and vapor (colloid-poor, V) phases. (b) The logarithm of the reduced surface tension $\beta \gamma \sigma_{C}^{2}$ plotted as a function of the logarithm of the relative distance to the critical point in terms of the pressure difference, $\left(P-P^{(c r)}\right) / P^{(c r)}$.

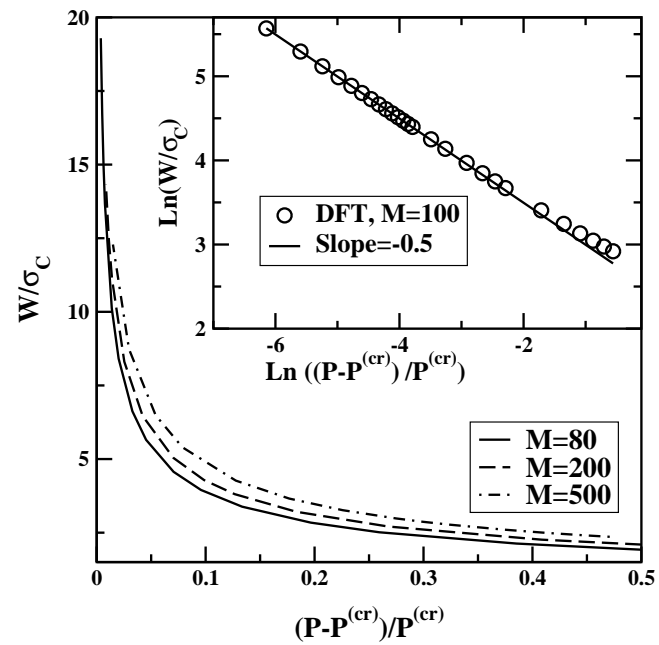

FIG. 6: The interfacial width $W$ as a function of the relative distance to the critical point expressed in terms of the pressure difference $\left(P-P^{(c r)}\right) / P^{(c r)}$. The main plot shows the interfacial width calculated for $M=80$ (solid line), 200 (dashed line) and 500 (dot-dashed line). The inset shows a double-logarithmic plot of the same quantities evaluated for $M=100$. The open circles denote the DFT results whereas the solid lines have slopes corresponding to the classical (mean-field) critical exponents. 


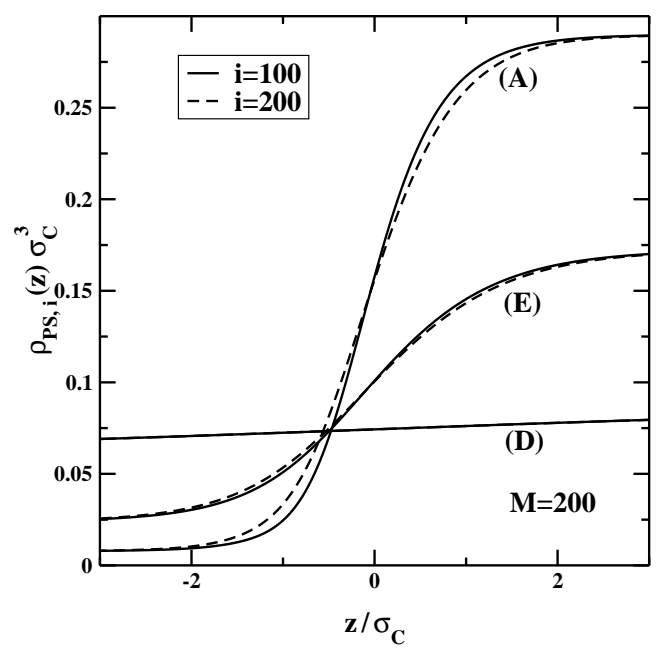

FIG. 7: Middle (the solid lines) and end (the dashed lines) segment density profiles across the liquid-liquid interface calculated for the system with $d=10$ and $M=200$. The profiles are evaluated for the reduced pressures $\beta P \sigma_{C}^{3}=7(\mathrm{~A}), 4.5(\mathrm{E})$ and 3.58 (D).

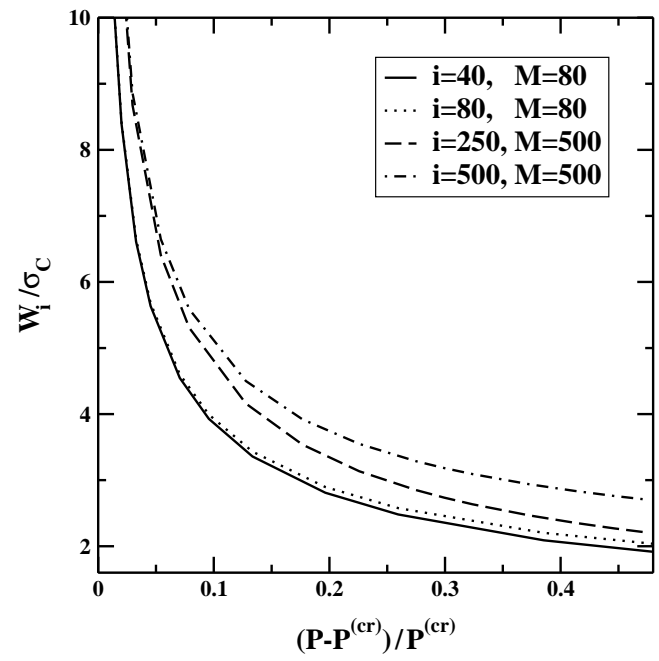

FIG. 8: The interfacial width of the segment density profile $W_{i}$ as a function of the relative distance to the critical point expressed in terms of the pressure difference $\left(P-P^{(c r)}\right) / P^{(c r)}$. The solid and dotted lines denote the interfacial width of the mid $(i=40)$ and end $(i=80)$ segment density profile for 80-mer whereas the dashed and dot-dashed lines denote the interfacial width of the mid $(i=250)$ and end $(i=500)$ segment density profile for 500-mer. 